\title{
TBM Process Data Management System
}

\author{
P. Moreno, M. Ruiz, and F. J. Gorines
}

\begin{abstract}
Total control of a tunnel project is currently possible with intelligent self-monitoring tools. The implementation of operational parameters of the TBM with other related issues around the construction process provides high quality information useful for the performance improvement. In this paper, we present a novel and breakthrough data management platform developed by ACCIONA Infrastructures aimed to be applied in mechanized tunneling: the "TBM Control data Center (TCC)". On-going monitoring, powerful data acquisition, remote management, failures surveillance and adaptive operation behavior are some of the main benefits of the TCC system.
\end{abstract}

Index Terms-Data management, intelligent tunneling, real-time monitoring, TBM.

\section{INTRODUCTION}

Tunnel construction projects exhibit a high amount of risks affecting the time and costs targets. The geological nature of the excavated media release added uncertainties to tunneling that other kind of engineering fields don't provide. Furthermore, when tunneling is developed by means of mechanized techniques like TBMs the construction process should became very complex (see Fig. 1) because of the high amount of information from both, geology and machine operation [1]-[3].

Nowadays, the majority of the TBM manufacturers are implementing its own data acquisition and parameter control systems. Those systems are able to compile detailed information about the TBM driving, excavation, lining installation, ground control measures, and logistics and so on. In spite of its potential to ease and improve the tunneling development, those systems are not able yet to solve specific issues affecting the whole tunneling process since:

- To make this high amount of raw information useful, it is necessary to make great efforts by the technical staff on site to grant an effective TBM monitoring and frequently, time is not always available.

- Information must be continuously filtered depending on the needs of Clients, Contractors or Suppliers. They tend to requires different kind of information and specific data which the other don't need or should not have.

- But the main problem that conventional data acquisition systems can't be solve is the implementation of the

Manuscript received April 25, 2014; revised June 29, 2014. This work was supported in part by the U.S. Department of Commerce under Grant BS123456.

The authors are with the ACCIONA Infraestructuras, S.A. Technology Innovation Department, Alcobendas, Madrid, CP 28108 Spain (e-mail: patricio.moreno.montero@acciona.com, manuel.ruiz.calvo@acciona.com, franciscojavier.gorines.garnacho@acciona.com). geological information within the construction cycle, therefore the relationships between operation parameters and the excavated ground can't be properly correlated

- ACCIONA Infrastructures as a result of its innovative culture and applied research in underground technology, the development of a novel monitoring system of TBMs has been recently released: the TCC (TBM Control data Center). The new platform tends to fill the gap left by the conventional data acquisition systems provided by TBM manufacturers, allow giving one step ahead during operations monitoring.

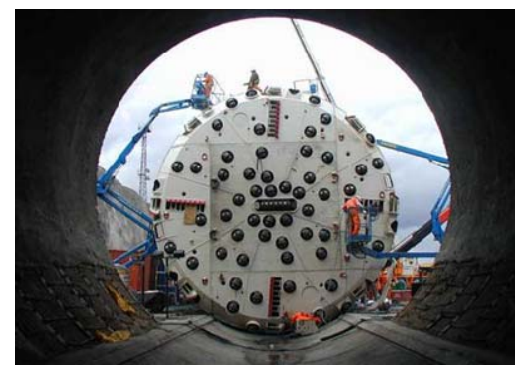

Fig. 1. TBM assembly.

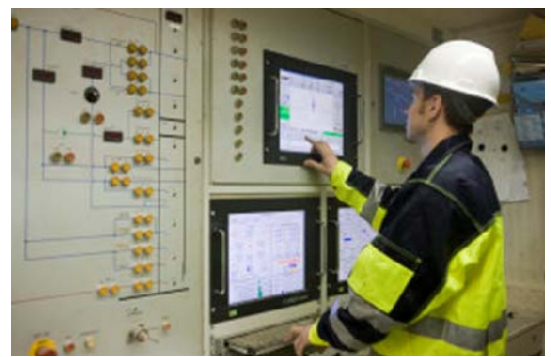

Fig. 2. TBM control console.

The TCC system tends to be more than a self-monitoring tool since it makes it possible the implementation in a single management system of all the construction processes involved during tunneling. Some of the main features that can be managed thanks to the TCC are:

- Operation parameters: torques, thrusts, penetration rates, cutter-head speed, injection pressures, chamber pressures...

- Geology: register of the rock mass quality, lithological description or geotechnical properties by means the integration of the project geological profile, by geological description of the excavated material or implementing laboratory information.

- Machinery maintenance: cutter wears, additive consumptions, failures and repairs

- Logistics and quality: surveillance of lining segments stock and installation, traceability, etc...

In the following sections we will present a brief summary 
of the TCC platform regarding its connectivity layout, the user interface, the main benefits provided by the system as well as a practical case history where the TCC has been successfully implemented.

\section{SYSTEM OVERVIEW}

The minimization of undesirable situations while tunneling becomes crucial when construction methods addressed the use of TBMs. The complexity and low tolerance for error (especially in urban underground environments) makes tunneling a highly potential monitoring process. Modern TBMs are well instrumented machinery capable of recording large sets of operational data related with excavation, lining installation, power demand, backup logistics...[4].

In this sense, the development and affordability of sensing and computation technologies have led to significant advances in sensing, data acquisition and real-time monitoring systems [5]. On this way, it can highlight some of most relevant and modern of these applications (without prejudice to other manufacturers): CAT ${ }^{\circledR}$ Tunnel Link software (Caterpillar Tunneling Canada Corporation); $\mathrm{MH}$ Drill Viewer (Aker Wirth); or Disc Cutter Rotation Monitoring, DCRM (Herrenknecht AG). All these applications are supplemented in the use of web platforms that will allow access to information stored on servers in real time.

Otherwise, a set of information management systems have been developed [6], [7]. This type of systems regard to improve relationships machine-user and easing the information management by the staff on site with the aid of statistical information, production reports or any other filtered operation information related with TBM. Examples as IRIS or TPC highlight because of its widespread use [8], [9]. However, those systems are not able to join into a whole, all factors affecting the TBM process, starting by the geology issue and following by the safety and quality control.

\section{TBM CONTROL DATA CENTER By ACCIONA}

Taking care the limitations of the current management systems ACCIONA Infrastructures TCC aims to cover those deficiencies by means the implementation of the following advances:

- Implementation of geology information within the whole tunneling process.

- Integration of external TBM processes, quality and logistics.

- Ensuring the persistence on the storage of raw data.

- Information access based on roles.

- Own and friendly graphical display of data collected.

- Ability to send alarms to the appropriate agents when certain parameters exceed the thresholds.

- Implementation of predictable algorithms.

- Full adaptability and transferable to any commercial acquisition data.

The development of TCC aims to build a storage platform developing and remote monitoring of TBM that works in parallel with the systems provided by manufacturers of machinery, and try to optimize the work relying on following four features:

- Manage the information: in order to obtain a gathering drilling data on real time, centralization of data received, creation a database with all the data stored from executed works that allow perform an automation of process, make simulations of drilling and create prediction models to prevent failures during construction.

- Optimize personnel costs. Centralized database involve an adjustment of technical staff; minor personnel can manage several works in different places. Centralization of staff, together with the receipt data on real time, brings a better support in less time.

- Reduce maintenance efforts and optimization of the TBM reparation schedule by means the gathering the information flow between machinery, workshop and production department.

- Consequently, the reduction the dependence of the manufacturer.

Operational parameters measured while tunneling (include torque of the cutterhead, axial force and displacement in each thrust jack, cutterhead RPM, and face pressure. These data are typically collected with the machine's central control system programmable logic controller (PLC).

During the excavation, the raw data are received from different sensors arranged in TBM on real- time (see Fig. 2). Each machine and/or manufacturer send the information in a different way, thus it must be necessary to create a common language.

The system developed tries to bridge the differences between machine types and machine data acquisition systems. In order to do this, the system works in a common architecture, storage system and interface.

\section{System ARChiteCtURE}

The implementation of the TCC in the TBM involves the following hardware items (Fig. 3):

- PLC: digital electronic device with a programmable memory for storing instructions and perform logic functions configuration sequence, timing, counting and arithmetic to control machine and process.

- Optic fiber: the information read by PLC is moved through the fiber optic network to the local data manager.

- Local Data Manager: this box contains the acquisition data software and it is responsible for processing the data received and stored in the data server.

- Database server. This equipment will host the information recorded during tunneling. It shall contain all the information concerning to operation, geology, logistics and so on to be used in the public part of the system. Also, here will be installed the report server.

- Mirror database server. Here will be hold a replica of the database, so if the previous server has a problem, it will be replaced in a short time period, preventing 
information losses and ensuring the continuous data record.

- Email server: this server allows to send the information (charts, reports, alerts, etc.) to users and administrators.

- Web server: contains de Web application that allows remote reviewing of the information gathered by PLCs.

- End devices: finally remote access can be done by means of PC, Tablets, etc.
Summarizing, the whole platform architecture allows extracting, transferring, transforming and loading data and records generated while tunneling to a homogeneous format that can be stored and treated in a single information platform. Those entire infrastructures provide the required adaptability and openness desired in order to the implementation of the whole volume of the information.

\section{舶acciona}

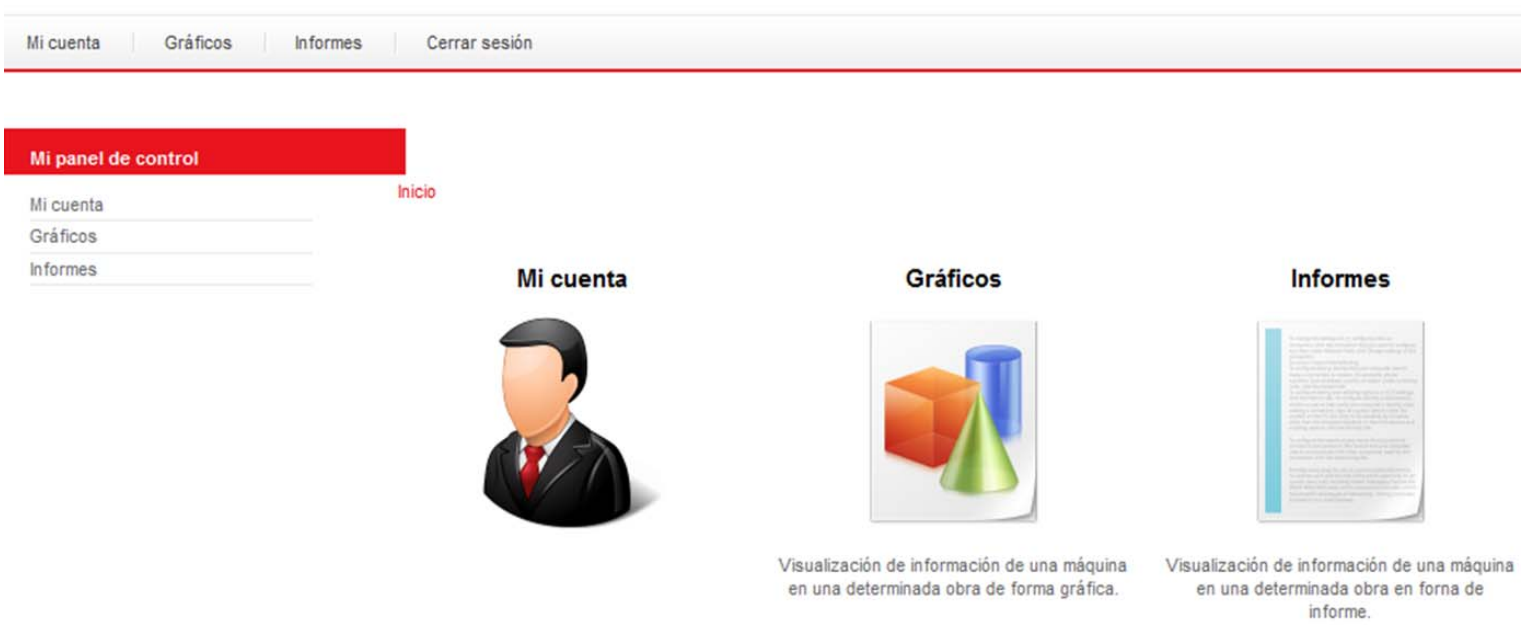

Fig. 4. Web app main menu overview.

\section{Data Storage and Migration Transference}

In order to store in an orderly and accessible manner the recorded data it is necessary to build a robust data base able to support the huge data flow generated. To do that, a Microsoft SQL Server was selected because of the following reasons:

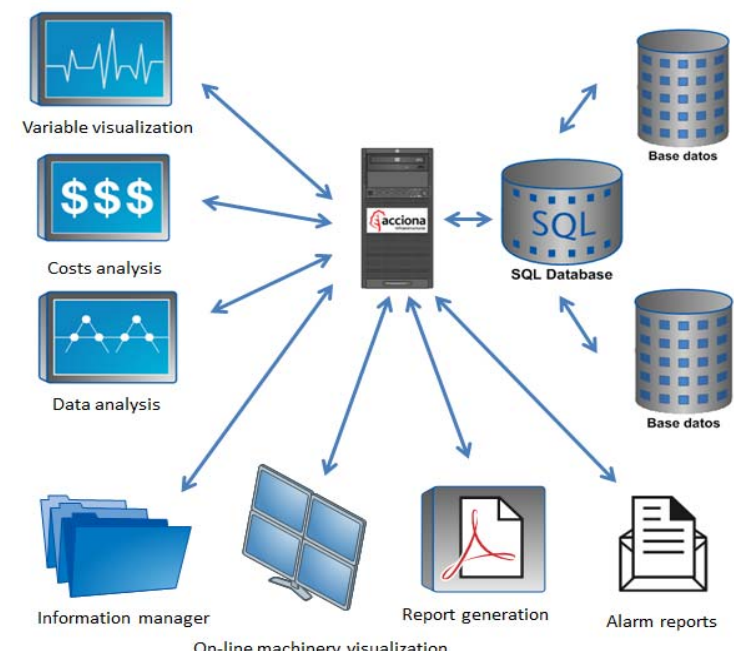

Fig. 3. System layout.

- Is a multi-user data system.

- It allows integrating in an easy way very flexible and comprehensive reports in many formats and via e-mail (see Fig. 4).

- It automates the replication of data between the main server and the mirror server so the data flow is always ensured. In spite of the backups strategy is useful to keep the information periodically, the occurrence of any server failure would produce information cut offs that backups can't avoid.

TBM provide the recorded data as DBF files associated to each installed lining ring. In those files, the data is recorded in a regular frequency interval (each $10 \mathrm{secs}$ ). Information about advance rates, construction cycle, comments, alarms and state are separately registered. Mean, first, last, maximum and minimum measured values during a single ring installation are collected.

In order to handle efficiently all the information produced by the different TBM acquisition systems TCC provides a tailored Data Loader tool which performs the migration of the data generated by the TBM to a single database.

This application is only enabled for Administrators.

\section{ADMINISTRATION AND USER INTERFACE}

The public interface of the platform implements a set of functions that allow users to view and manage the recorded information. In addition, a web application allows remote access to all the information produced by each TBMs worksite providing a friendly interface and efficient relationships machine-user.

A system data access authentication process based on roles is implemented. Two types of roles are clearly differenced: system administrators and basic users.

Administrators can easily perform the following operations by means the administration console: 
- Application configuration of: work sites, manufacturers, machine types, sensors, menu items.

- Account management: user management, role management, visualization privileges, data migration access.

- Output management

- Report management

The TCC system also includes a friendly interface called, TBM viewer (see Fig. 5) in order to control, in a simple glance, the general parameters during the tunneling process.

\section{PRACTICAL CONTRIBUTIONS}

The main competitive advantages, technical improvements and new concepts provided by the TCC are closely related with the capability to join the whole information of the project within the TBM operation process. In a simple way, the TCC offers the added value of an intelligent ITC tool experienced-based providing construction adaptability and cost and time reductions.

This tool exhibits a powerful usefulness for a tunnel contractor because the information can be managed in many different senses.

- Bid and Tender department: since the company has a deeply know how from the learning of previous projects development, it will be possible to give attractive and more competitive technical and economic solution to the Clients during the tender phases of new projects.
- Purchasing department: the wide experience in cost control, consumptions and suppliers from previous and present projects allow huge advantages while the purchase process. The optimization of materials availability, the reduction of costs in critical consumables and the selection or rejection of common suppliers are some of the main advantages related with purchase management.

- Machinery department: TBM behavior is constantly surveyed. The monitoring of the main hydraulic, electrical or mechanical parts involved during excavation, lining installation and gap injection allows identifying weaknesses of the machinery. It will allow quick communication with the TBM manufacturer and the own machinery department in order to save money and to learn the better way to drive each specific machine and ground nature.

- Remote assistance: since the TCC allows a total remote control far away the TBM, specialist staff can give adequate support during tunneling. Unexpected problems will be regarded by TCC staff and stoppages and times properly reduced.

- Staff training: TCC provides an ideal framework to host training sessions to large groups of people since the sessions can be developed from the comfortable room of the TCC headquarter far away the reduced space and noisy pilot cabin.
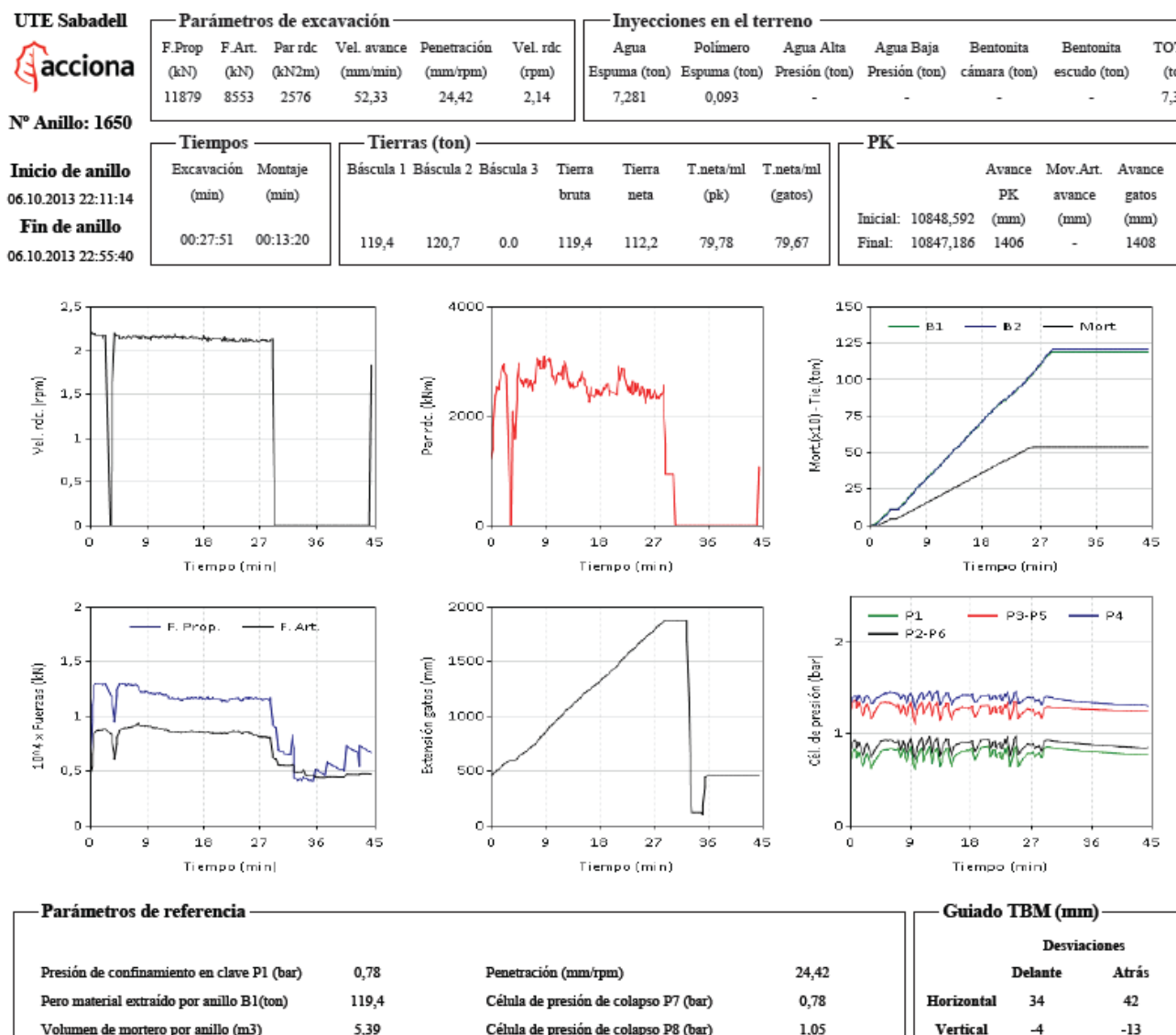

0,78
119,4
5,39

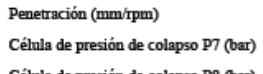

Célula de presión de colapso P8 (bar)
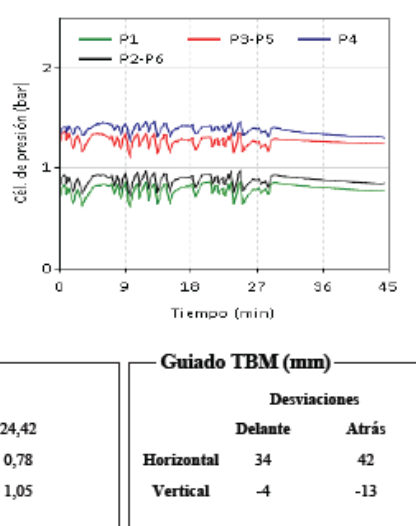
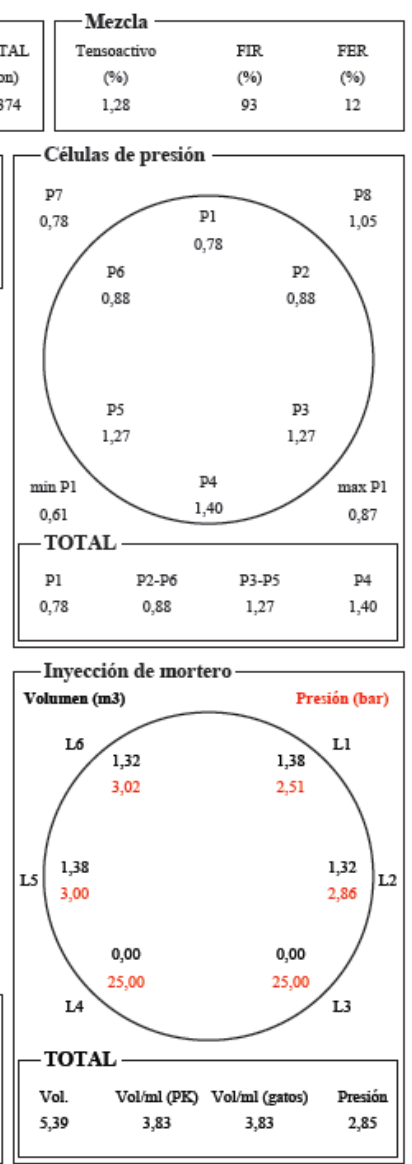

Fig. 5. TBM viewer. 

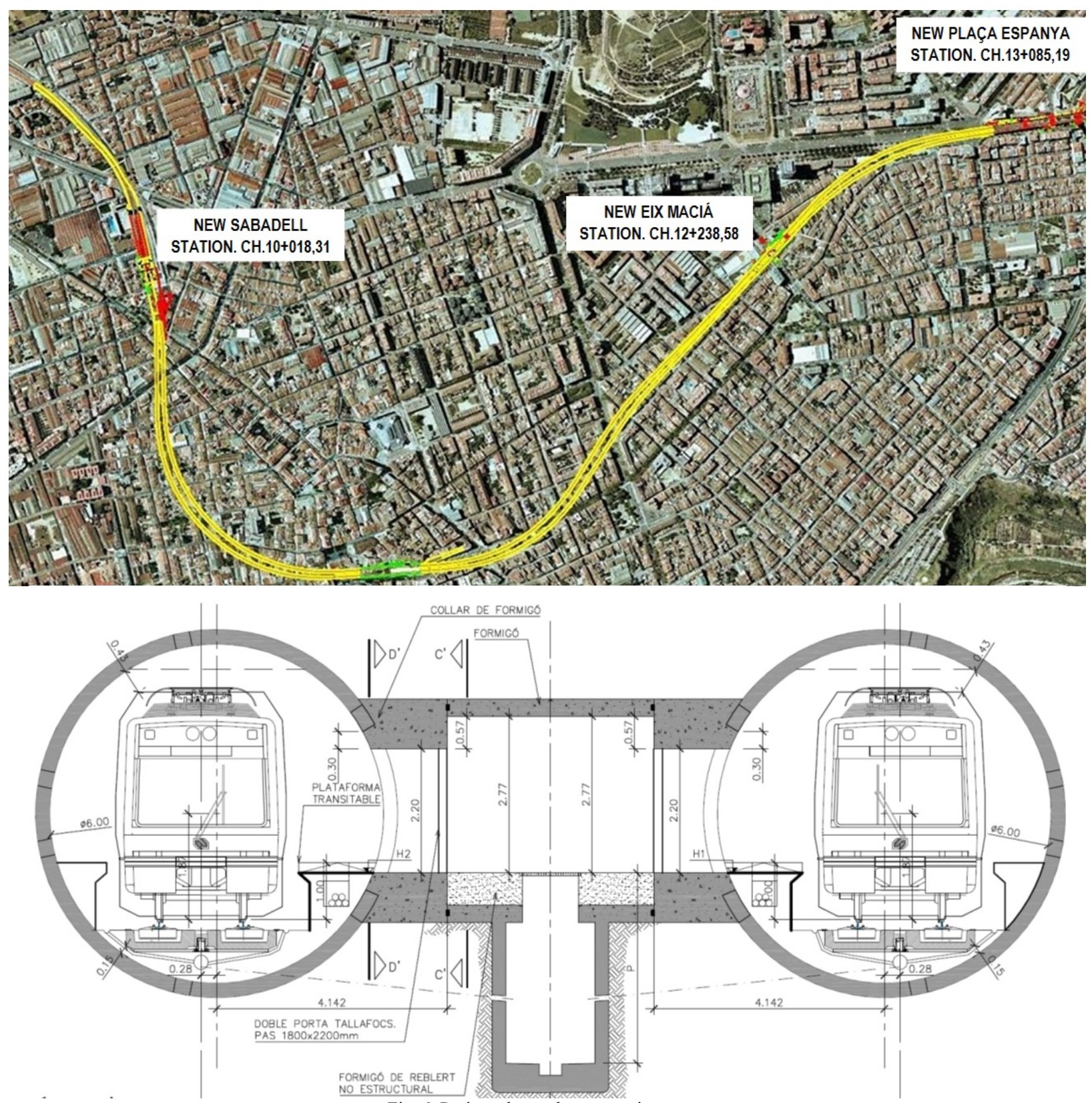

Fig. 6. Project plan and cross section.

\section{A Real Case: Suburban RaIlway of Sabadell}

ACCIONA Infrastructures TCC has been tested with success in some recent projects during the construction of the HSRL Madrid-Asturias (Pajares Tunnels 1 and 5 sectors) and the HSRL Atlantic Axis (Das Maceiras Tunnel) among others. Here, we will present the experience during the construction of the Suburban Railway Extension to Sabadell (Spain).

The project, consisting on the construction of two twin single track tunnels of approximately $3 \mathrm{~km}$ length (each one), was developed by a Spanish joint venture led by ACCIONA Infrastructures and guided by AMBERG Infrastructures (see plan and cross sections in Fig. 6). A 6 meters diameter EPB (Earth Pressure Balance) machine was specifically built to this project by Herrenchneckt (Fig. 7). Both tunnels were recently successfully finished, reaching advance rates peaks up to $60 \mathrm{~m} /$ day.

Various operational problems emerged during the project development and the TCC system provides useful solutions in order to manage them properly.

One important benefit of the construction of two parallel tunnels is the possibility to make accuracy comparisons between both tubes. In this case the TCC becomes very useful to give ground behavior comparisons to keep adequate safety conditions and high productivity. In this sense the following issues could be addressed:

- Information about foam, resins or mortars consumptions allows estimating geology changes anticipating undesirable situations and making better geological accommodations.

- The settlement measures collected during the previous tube allowed the optimization of the operational parameters while tunneling near buildings and other critical situations, reducing risks and increasing performance.

- CCT has also proved useful for supplies management. Since the necessities on additives storage are close related with geology demand, the system was able to 
predict storage necessities and purchase strategies as well.

- The logistic control over the volume of excavated material provides a refined control over the earth works suppliers, specifically on the transportation of the tailings to landfill.

- Also, those measures allow to control over excavations and excesses of mortar volumes during gap injection. Comparisons were possible and the operation cycle was properly improved.

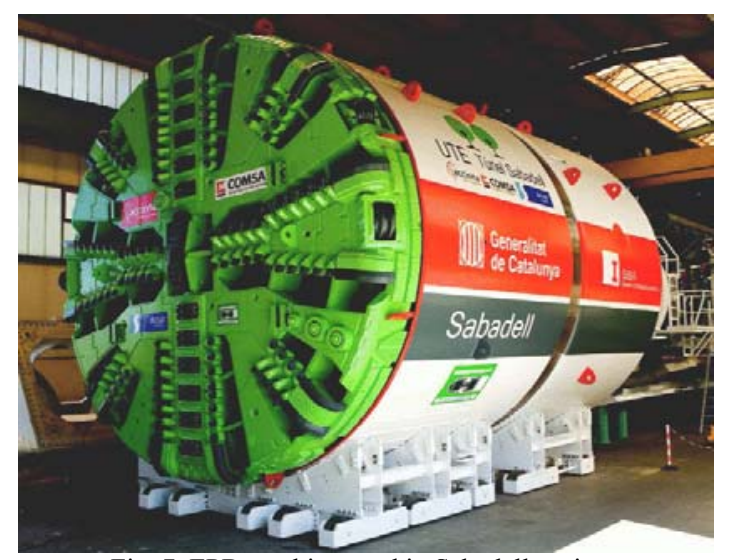

Fig. 7. EPB machine used in Sabadell project.

The experience in the previous tube during the Sabadell project development provides an intelligent TCC feedback on the new twin tube. The similarities between both, left and right tube, allow high robustness and replicability so a better understanding of the TBM operation behavior was possible. As the reader can observes TCC has been able to implement in a single management platform the whole information from TBM operation, geology, quality control, logistics and maintenance, therefore the integration of the total production control was react.

The TCC was able to analyze more than 65.530 data points per 250 milisecs thanks to the implementation of optic fiber for data communication. As a result of the analysis of thousands of data points during EPB operation it was possible to balance the excavation parameters within the optimum working range, making compatible a high excavation performance, quality levels and safety environments with minimum operation costs.

Sabadell project is a practical example of how ACCIONA Infrastructure TCC ease the EPB operation and maintenance in real-time by means the own self-monitoring and information compilation. The main impacts of the TCC in the Sabadell project were:

- Reduction of $20 \%$ of production personnel costs.

- Reduction of $15 \%$ of TBM maintenance costs.

- Reduction of $10 \%$ the time target with a corresponding operation cost reduction.

\section{Final COMments AND CONCLUSIONS}

Tunnelling projects developed by means TBMs require the minimization of risks associated to geology changes since the construction method are inflexible and highly routine. It is widely accepted that TBM operation are close related with the ground properties and its behaviour so the parameter control is highly recommended in order to get success scenarios and meet targets.

The risk minimization and the reduction of uncertainties as well are key topics in modern underground projects to get cost-efficiency and highly safety environments. In this sense, the high amount of data provided by the current acquisition systems implemented in the TBMs is very useful and potentially powerful. However, the lack of the ability to implement all the issues involved while tunnelling (mainly geology but also quality, costs control, materials supply or safety) makes the conventional acquisition systems a simple provider tool of raw information.

In order to develop integrating tools to answer the limitations explained above ACCIONA Infrastructure has developed the TBM Control data Center (TCC) system. This tool allows the following improvements within the current technology:

1) Geology implementation within the whole operation process.

2) Total peripheral issues implementation (quality, safety, costs, personnel, schedules...)

3) Ability to store the whole information and get continuous know how.

4) Intelligence skills due to the implementation of algorithms able to recognize previous experience and therefore, adaptive behavior is possible.

5) Independence from the machine manufacturer.

6) Real-time monitoring with high speed rates of data acquisition.

7) Robust and stable information management.

8) Remote control and efficient aid support.

In a summary, TCC system states a novel breakthrough ITC solution for tunneling management able to ease the daily tasks of the personnel on site as well as to improve TBM performance and minimizing time and costs. TCC system makes real the total implementation of the construction parameters in a single and friendly use platform, providing a step ahead within the state of the art by means the fusion of construction engineering with ITCs.

\section{ACKNOWLEDGMENT}

Author wish specially thanks Mr. José M. Creus (Department Director at ACCIONA Infrastructures and General Manager of Sabadell JV) and Mr. Pablo Castro (Tunnel Manager of Sabadell JV) the efforts done in order to make real the TCC project. Also, the contribution of the Technology and Innovation Division of ACCIONA Infrastructure staff to host the project and to enhance the technology transference.

\section{REFERENCES}

[1] V. Guglielmetti. "Process Control in Mechanized urban tunneling," Mechanized Tunneling in Urban Areas. Design Methodology and Construction Control, 2007, p. 507.

[2] V. Marchinni and V. Guglielmetti. "EPB - Tunnelling control and monitoring in an urban environment: the experience of the "Nodo di Bologna," construction (Italian High Speed Railway System)," Underground Space-Dimensions of Metropolies, 2007, pp. 985-901.

[3] M. A. Mooney, B. Walter, and C. Frenzel, "Real-time tunnel boring machine monitoring: A state of the art review," North American Tunnelling, 2012, pp. 73-81. 
[4] M. Marchioni, A. Selleri, F. Stahl, and M. Messina, "Intensive application of the TBM data management system for the work supervisor of the largest worldwide TBM-EPB project," World Tunnel Congress Geneva Underground, 2013.

[5] S. Sumitro, T. Okamoto, and D. Inaudi, "Intelligent sensory technology for health monitoring based maintenance of infrastructures," Smart Sensor Technology and Measurement Systems, SPIE, 5384-19, San Diego, California, 2004.

[6] J. M. Donald, C. Dishlip, and P. C. Raleigh, "Tunnelling data: turning information into intelligence on the NEIS project," RETC Proceedings, pp $1183-1191,2005$.

[7] P. Thurlow. "Supporting measures for tunneling," in Proc. $8^{\text {th }}$ International Symposium on Field Measurements in GeoMechanics, 2011.

[8] Babendererde Engineers.
http://www.babeng.com/software. http://www.babeng.com/software.

[9] ITC ENGINEERS. http://www.itc-engineering.de/en/products/iris/iristunnel/

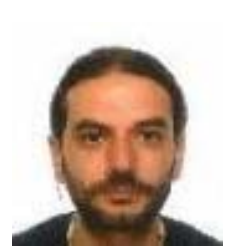

Moreno-Montero Patricio is currently working in the ICT R\&D group at the Technological Center of ACCIONA Infrastructures in Madrid (Spain). While working in this group he implemented over 20 instances of a document management system as well as a biometric access control system for constructions sites (currently being implemented).

$\mathrm{He}$ also participated in the European projects CESAR (Cost-efficient methods and processes for safety relevant embedded systems), Fiemser (Friendly Intelligent Energy Management Systems in Residential Buildings) and make Sense (Easy Programming of Integrated Wireless Sensor Networks). In this last project, he published, among other authors, the paper "Towards business processes orchestrating the physical enterprise with wireless sensor networks". Currently his research is focused on the use of artificial vision in construction sites. He is a PC member of the 8th System Analysis and Modelling Conference (SAM 2014). 